\title{
ARMAND BOREL
}

The accompanying articles shows some of the many sides of Armand Borel, who died August 11, 2003. The eight authors write about him in the order Serre, Chandrasekharan, Bombieri, Hirzebruch, Springer, Tits, Arthur, Prasad.

Borel's research in algebra and topology was good enough to get him appointed professor at the Institute for Advanced Study at age thirty-four. Serre gives an overview of the mathematics, and Hirzebruch describes that mathematics in a different way, with emphasis on the topology. Springer and Tits write about Borel's work in algebraic groups, and Arthur writes about arithmetic groups and about how Borel's work in this area laid the foundations of the modern theory of automorphic forms.

An Institute colleague said that Borel believed strongly in the unity of mathematics and in the importance of the written record. The means for acting on these beliefs included roles as editor, author, educator, and conference organizer, and some of the present authors have elaborated on these activities. Borel put a sizable effort into contributions to the volumes by Bourbaki, detailing his experiences with that group in an article "Twenty-five Years with Nicolas Bourbaki, 1949-1973" in the Notices in March 1998 [E 165]. He is widely regarded as having played a major role in the writing of the Bourbaki chapters on Lie groups and Lie algebras, which have been of particularly enduring value.

Borel was an editor of Annals of Mathematics for 1962-79 and of Inventiones Mathematicae for 1979-93, among other journals. For the interval 1998-2000 he served quietly as a kind of unofficial associate editor for the Notices, advising the editor on various matters, particularly memorial articles and the collaboration of the Notices of the American Mathematical Society with its counterparts in other countries. Borel played a large but anonymous role in planning the various articles about A. Weil and the memorial articles for J. Leray and A. Lichnerowicz.

Some of the authors of the accompanying articles describe some of the books that Borel authored or edited. The list of such books has seventeen entries, apart from his Euvres: Collected Papers [E], and appears at the end of this article. A number of these books are outgrowths of seminars, sometimes joint with other people and sometimes not. No matter what form the seminars took, one can be confident that Borel was the choreographer of each. Of special note are the proceedings from two AMS summer institutes, in Boulder [3] in 1965 and in Corvallis [10] in 1977. Each proceedings contains significant expositions by Borel and contributions by many other experts; each has become a basic reference in its field.

In his last few years Borel kept up an annual schedule at the Institute in the winter, the Far East in the spring, and Switzerland in the summer. For each year 1999-2001 he was the organizer of a program on Lie theory at Hong Kong University from March to July. He had planned a summer school at Zhejiang University in Hangzhou, China, with Lizhen Ji and S.-T. Yau for August 2003, with continuations

Reprinted from the Notices of the AMS (Volume 51, Number 5) with the permission of the American Mathematical Society.

Department of Mathematics, State University of New York, Stony Brook, NY 11794-3651, USA (aknapp@math.sunysb.edu). 
in 2004 and 2005 and plans for publishing the proceedings. The summer school for 2003 took place as planned, but Borel was unable to attend.

Borel was a counselor to mathematicians young and old. He had a fearsome reputation, and making a first approach to him was not for the faint-hearted. Yet many stories have come out since his death about how he helped individual mathematicians in large and small ways. All a person had to do was ask, and suddenly the effect of Borel's personality completely changed. Prasad writes how responsive Borel was to inquiries about mathematics from anyone anywhere in the world.

How did this man maintain his creative spirit, his energy, and his enthusiasm for so long? His older daughter, Dominique, said of him that he approached each new thing in his life, mathematical or otherwise, with the attitude of wonder and excitement of a small child. The citation for his AMS Steele Prize for Lifetime Achievement, which he was awarded in 1991, concluded with the following comments on Borel's activities beyond research: "In the course of amassing these astounding achievements, he placed the facilities of the Institute for Advanced Study at the service of mathematics and mathematicians, using them to foster talent, share his ideas, and facilitate access to recent developments through seminars and lectures. It is just simply not possible to cite a career more accomplished or fruitful or one more meaningful to the contemporary mathematical community."

\section{Books by Armand Borel.}

[E] Euvres: Collected Papers, Springer-Verlag, Berlin; vol. I, II, III, 1983; vol. IV, 2001.

[1] Cohomologie des Espaces Localement Compacts, d'après J. Leray, École Polytechnique Fédérale, Zürich, 1953; 2nd edition, 1957; 3rd edition, Lecture Notes in Math., vol. 2, Springer-Verlag, Berlin, 1964.

[2] Seminar on Transformation Groups, Annals of Math. Stud., vol. 46, Princeton University Press, Princeton, NJ, 1960.

[3] (Edited with G. D. Mostow) Algebraic Groups and Discontinuous Subgroups, Proc. Sympos. Pure Math., vol. IX, American Mathematical Society, Providence, RI, 1966.

[4] (Written with S. Chowla, C. S. Herz, K. Iwasawa, J-P. Serre) Seminar on Complex Multiplication, Lecture Notes in Math., vol. 21, Springer-Verlag, Berlin, 1966.

[5] Topics in the Homology Theory of Fibre Bundles, Lecture Notes in Math., vol. 36, Springer-Verlag, Berlin, 1967.

[6] Introduction aux groupes arithmétiques, Actualités Sci. Indust., vol. 1341, Hermann, Paris, 1969.

[7] Linear Algebraic Groups, W. A. Benjamin, Inc., New York, 1969; 2nd edition, Grad. Texts Math., vol. 126, Springer-Verlag, New York, 1991.

[8] (Written with R. Carter, C. W. Curtis, N. Iwahori, T. A. Springer, R. Steinberg) Seminar on Algebraic Groups and Related Finite Groups, Lecture Notes in Math., vol. 131, Springer-Verlag, Berlin, 1970.

[9] Représentations de Groupes Localement Compacts, Lecture Notes in Math., vol. 276, Springer-Verlag, Berlin, 1972.

[10] (Edited with W. Casselman) Automorphic Forms, Representations and LFunctions, Proc. Sympos. Pure Math., vol. XXXIII, Parts I and II, American Mathematical Society, Providence, RI, 1979.

[11] (Written with N. R. Wallach) Continuous Cohomology, Discrete Subgroups, and Representations of Reductive Groups, Ann. of Math. Stud., vol. 94, Princeton 
University Press, Princeton, NJ, and University of Tokyo Press, Tokyo, 1980; 2nd edition, Math. Surveys Monogr., vol. 67, American Mathematical Society, Providence, RI, 2000.

[12] (Written with P.-P. Grivel, B. Kaup, A. Haefliger, B. Malgrange, F. Ehlers) Algebraic D-Modules, Perspect. Math., vol. 2, Academic Press, Inc., Boston, MA, 1987.

[13] Automorphic Forms on $\mathrm{SL}_{2}(\mathrm{R})$, Cambridge Tracts Math., vol. 130, Cambridge University Press, Cambridge, 1997.

[14] Semisimple Groups and Riemannian Symmetric Spaces, Texts Read. Math., vol. 16, Hindustan Book Agency, New Delhi, 1998, distributed by American Mathematical Society, Providence, RI.

[15] Essays in the History of Lie Groups and Algebraic Groups, History Math., vol. 21, American Mathematical Society, Providence, RI, and London Mathematical Society, Cambridge, 2001.

[16] (Written with R. Friedman and J. W. Morgan) Almost Commuting Elements in Compact Lie Groups, Mem. Amer. Math. Soc., vol. 157, American Mathematical Society, Providence, RI, 2002.

[17] (Written with Lizhen Ji) Compactifications of Symmetric and Locally Symmetric Spaces, Birkhäuser, Boston, to appear. 
A. KNAPP 